\title{
Voltammetric behaviour of oligonucleotide lipoplexes adsorbed onto glassy carbon electrodes
}

\author{
J.A.P. Piedade ${ }^{a}$, M. Mano ${ }^{\text {b,c }}$, M.C. Pedroso de Lima ${ }^{\text {b,c }}$, \\ T.S. Oretskaya ${ }^{\mathrm{d}}$, A.M. Oliveira-Brett ${ }^{\mathrm{a}, *}$ \\ a Departamento de Química, Universidade de Coimbra, Coimbra 3004-535, Portugal \\ ${ }^{\mathrm{b}}$ Departamento de Bioquímica, Faculdade de Ciências e Tecnologia, Universidade de Coimbra, Coimbra 3004-535, Portugal \\ ${ }^{\mathrm{c}}$ Centro de Neurociências e Biologia Celular, Universidade de Coimbra, Coimbra 3004-535, Portugal \\ ${ }^{\mathrm{d}}$ Chemistry Department, Moscow State University, Vorobjovy Gory, Moscow 119899, Russia
}

Received 16 June 2003; received in revised form 16 September 2003; accepted 6 October 2003

\begin{abstract}
The voltammetric behaviour of oligonucleotide lipoplexes (ODN-lipoplexes) prepared from short oligodeoxynucleotides (ODN), with different base compositions, and liposomes of the cationic lipid DOTAP, was studied by differential pulse voltammetry with a glassy carbon mini-electrode. It was found that the ODN base composition influences the ODN-lipoplex voltammetric response. Differential pulse voltammograms for ODN-lipoplexes of the ODN adenosine nucleotides present two different features when compared with the differential pulse voltammograms obtained for free ODN: a new peak appeared and the peak attributed to oxidation of adenosine diminished or was absent, depending on whether the ODN sequence had guanosine nucleotides or not. The presence of guanosine nucleotides in the ODN-lipoplex led to a peak due to guanosine oxidation with similar potential and current to the peak obtained for guanosine oxidation in free ODN. No detectable peaks were recorded in the voltammograms obtained with lipoplexes composed of ODN containing only pyrimidine bases. It was possible to show by voltammetry the occurrence of partial denaturation of short double helices of ODN when mixed with DOTAP liposomes to generate lipoplexes. The extent of denaturation was observed to increase with lipoplex $(+/-)$ charge ratio as shown by the increase in the differential pulse voltammetry peak currents. The electrochemical characterisation of lipoplex properties at a charged interface can be important for understanding and development of these gene therapy vectors.
\end{abstract}

(C) 2003 Elsevier B.V. All rights reserved.

Keywords: Voltammetry; Electrified interface; Lipoplex; Cationic liposome; DOTAP; Oligonucleotide

\section{Introduction}

Complexes between short oligodeoxynucleotides (ODNs) and liposomes containing cationic lipids, generally termed ODN lipoplexes, have been used in important nonviral vectors for gene delivery used in genetic therapy as sequence specific regulators of gene expression and anti-sense based therapeutics [1-4].

For the effectiveness of gene delivery into target cells, several factors must be considered concerning the stability of the lipoplex as it is important to protect them

\footnotetext{
${ }^{*}$ Corresponding author. Tel./fax: +351-239-835295.

E-mail address: brett@ci.uc.pt (A.M. Oliveira-Brett).
}

from nuclease activity, to assure their cellular uptake and improve ODN expression [1-5].

Among the factors that affect lipoplex stability, the nucleotide sequence and composition have been recently reported to influence some physicochemical properties involved in charge neutralisation and aggregation that occurs during lipoplex generation [6-9]. Nevertheless, a full characterisation of the interaction between ODNs and cationic liposomes is still required in order to understand how the base sequence and composition affects the lipoplex properties.

The voltammetry of ODN lipoplexes, composed by short ODNs containing only guanosine and cytidine nucleotides has been studied [10]. DOTAP (1,2-dioleoyl-3-trimethylammonium propane), a monovalent 
positively charged non-natural lipid that exhibits low toxicity in vivo (Scheme 1) [4], was used to generate cationic liposomes. It was verified that the peak current observed in the voltammograms obtained with ODN lipoplexes was due to guanosine oxidation and that the current was influenced by the adsorption potential used to adsorb ODN lipoplexes onto the electrode surface and by the ODN lipoplex $(+/-)$ charge ratio. It was found that for low $(+/-)$ charge ratios the lipoplex peak current obtained was enhanced when compared to that obtained for the same concentration with free ODN. This led to higher sensitivity in the determination of ODN containing guanosine nucleotides by differential pulse voltammetry. For ODN lipoplexes of unit molar (+/-) charge ratio the peak current obtained was very similar to that obtained for the same concentration with free ODN. This indicates that the same amount of oligonucleotide present in both situations was adsorbed on the electrode surface and able to undergo oxidation [10].

Based on the voltammetric data presented, a model was proposed to explain the reorganisation of ODN lipoplexes at the electrode|solution interface [10] that involves a transition between the liposome surface covered with oligonucleotides in solution, [8], to a sandwich-like configuration when ODN lipoplexes were adsorbed onto the electrode surface with applied potential, Scheme 2. Although there is no information on ODN lipoplex structure at a charged surface, the proposed model fits well with the experimental voltammetric data obtained.

The principal aim of the present work was the voltammetric characterisation of ODN lipoplexes using ODN sequences containing all DNA bases and to ex-

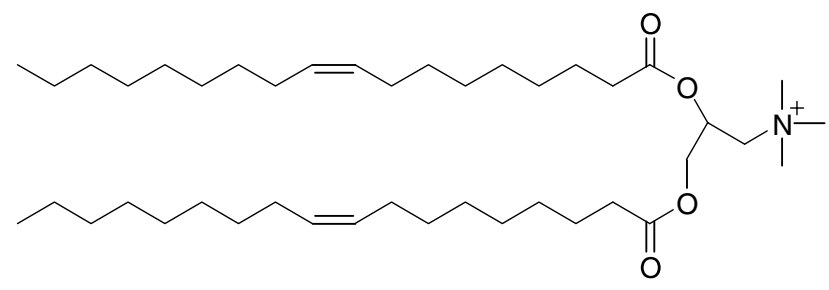

Scheme 1. Molecular structure of DOTAP. amine the influence of ODN base composition on the voltammetric response. The effect of lipoplexes on short ODN hybrids was also studied and the voltammetric results are presented and discussed.

\section{Experimental}

\subsection{Material and reagents}

Analytical grade reagents and purified water from a Millipore Milli-Q system (conductivity $<0.1 \mu \mathrm{S} \mathrm{cm} \mathrm{cm}^{-1}$ ) were used for the preparation of phosphate buffer electrolyte solution. DOTAP (1,2-dioleoyl-3-trimethylammonium propane) $25 \mathrm{mg} / \mathrm{ml}$ in chloroform solution was purchased from Avanti Polar Lipids (Alabaster, AL) and used without any further purification. The chloride anion was the DOTAP counterion.

The oligodeoxynucleotides (ODNs) used in this study are listed in Table 1 and were synthesised on an Applied Biosystems 380B automated DNA synthesizer (USA) using reagents for oligonucleotide chemistry purchased from Fluka (Germany). Buffered solutions of each ODN were prepared by diluting a certain volume of the ODN aqueous stock solution to obtain the desired concentration.

Nano- and microvolumes were measured using an EP-10 Plus and a EP-100 Plus Motorized Microliter Pippette (Rainin Instrument Co. Inc., Woburn, USA). The $\mathrm{pH}$ measurements were carried out with a Crison Model micropH 2001 pH-meter using an Ingold combined glass electrode. All experiments were done at room temperature $\left(25 \pm 1{ }^{\circ} \mathrm{C}\right)$.

Table 1

Sequence of oligodeoxynucleotides used

\begin{tabular}{lll}
\hline & Sequences $\left(5^{\prime} \rightarrow 3^{\prime}\right)$ & $\mathrm{Abs}_{260}(\mathrm{ou} / \mu \mathrm{g})$ \\
\hline I & AAAAAAAAAA & 28 \\
II & AAAAGGAGAG & 44 \\
III & CTCTCCTTTT & 50 \\
SALM & ACCTTGCTGAAATTTTCCC & 45 \\
COMP & GGGAAAATTTCAGCAAGGT & 47 \\
\hline
\end{tabular}

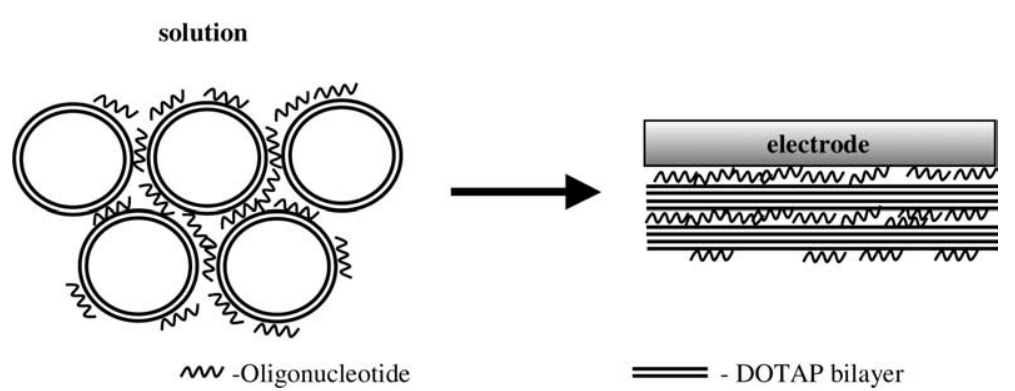

Scheme 2. Model for ODN lipoplexes in solution and attached to a positively charged electrode surface. 
2.2. ODN quantification, self-dimer formation and hybridisation

Concentrations of the aqueous solutions of ODNs were determined by measuring their absorbance at $260 \mathrm{~nm}$, considering the relation between optic units and $\mu \mathrm{g}$ for each sequence presented in Table 1. A Specord $\mathrm{S}$ 100, UV-Vis spectrophotometer (Carl Zeiss Technology, Analytic Jena, Jena, Germany) was used to measure the absorbance of the ODN solutions.

The extent and stability of any possible self-dimers and hairpin structures formed by the ODNs used were evaluated theoretically using the software PrimerSelect (DNASTAR, Inc.).

To generate hybrid ODNs, equal concentrations of stock aqueous solutions of complementary ODN strands were mixed together in $\mathrm{pH}$ 7.1 $0.1 \mathrm{M}$ phosphate buffer solution to the desired final concentration. The solution with the two complementary strands was heated to $65^{\circ} \mathrm{C}$ over $15 \mathrm{~min}$ and then allowed to cool to room temperature. Hybridisation was allowed to proceed for at least $12 \mathrm{~h}$ before the voltammetric assay or the addition of cationic liposomes to generate the correspondent lipoplexes with the $(+/-)$ charge ratio desired. In a control experiment the extent of hybridisation was monitored by the characteristic decrease in absorbance at $260 \mathrm{~nm}$ with time.

\subsection{Preparation of cationic liposomes}

For the preparation of DOTAP (1,2-dioleoyl-3trimethylammonium propane) liposomes [11], a dry film of $3 \mu \mathrm{mol}$ lipid was produced under vacuum using a rotatory evaporator from a stock solution in chloroform. The dried lipid film was then hydrated with distilled water to a lipid concentration of $3 \mu \mathrm{mol} / \mathrm{ml}$ and sonicated for $5 \mathrm{~min}$. The resulting liposomes were then extruded 21 times through two stacked polycarbonate membranes of $50 \mathrm{~nm}$ pore diameter using a Liposofast device (Avestin, Toronto, Canada), and stored at $4{ }^{\circ} \mathrm{C}$, under nitrogen, until use. Liposomes were used within one month after preparation.

\subsection{Lipoplex formation}

The complexes between ODN and DOTAP cationic liposomes, the ODN lipoplexes, were prepared at different DOTAP/ODN (+/-) charge ratios by gently mixing the phosphate buffered ODN solution with a fixed volume of the liposome stock solution, depending on the charge ratio desired. The mixture was left for $15 \mathrm{~min}$ at room temperature prior to the voltammetric experiment.

The ODN lipoplex charge ratio was calculated considering that $1 \mu \mathrm{mol}$ of cationic lipids corresponds to 1 $\mu \mathrm{mol}$ of positive charges and $1 \mathrm{mg}$ of ODN has 3.08 nmol of negatively charged phosphate groups at $\mathrm{pH} 7.1$.

\subsection{Voltammetric cell and parameters}

All voltammetric experiments were done using an $\mu$ Autolab Type II running with GPES version 4.9 software (Eco-Chemie, Utrecht, The Netherlands). A onecompartment electrochemical cell with a volumetric capacity of $100 \mu \mathrm{l}$ was used containing a $1.5 \mathrm{~mm}$ diameter glassy carbon mini-electrode (Cypress, USA), a $\mathrm{Pt}$ wire counter electrode, and an $\mathrm{Ag} \mid \mathrm{AgCl}$ reference electrode ( $3 \mathrm{M} \mathrm{KCl}$ saturated with $\mathrm{AgCl}$ ). This cell was placed inside a Faraday cage at room temperature during all the measurements.

The voltammetric parameters used, unless stated otherwise, were: differential pulse voltammetry, pulse amplitude $50 \mathrm{mV}$, pulse width $70 \mathrm{~ms}$, scan rate $5 \mathrm{mV} \mathrm{s}^{-1}$. All potentials are referred to $\mathrm{Ag} \mid \mathrm{AgCl}$.

\subsection{Pretreatment of the glassy carbon mini-electrode}

Before and between every electrochemical assay with samples of ODNs the glassy carbon mini-electrode was polished using alumina oxide particle size $0.3 \mu \mathrm{m}$. After polishing, it was rinsed thoroughly with Milli-Q water for $30 \mathrm{~s}$, the electrode was sonicated for $1 \mathrm{~min}$ in an ultrasound bath, and again rinsed with water. After this mechanical treatment the mini-electrode underwent electrochemical pre-treat that consisted of three steps: first, the electrode was scanned in the electrolyte solution to check the cleanliness of the electrode surface; second, a $+1.65 \mathrm{~V}$ potential was applied for $300 \mathrm{~s}$; and finally the electrode was scanned between +0.3 and +1.3 $\mathrm{V}$, until a steady-state baseline voltammogram was obtained. This electrochemical pre-treatment improves the currents recorded making the assay more sensitive and reproducible [12], and so was employed in all the voltammetric experiments presented.

\subsection{Acquisition and presentation of voltammetric data}

After the electrochemical pre-treatment the minielectrode was immersed in the sample solution and a $-0.1 \mathrm{~V}$ adsorption potential was applied for $300 \mathrm{~s}$. The mini-electrode with the adsorbed sample was then gently washed with deionised water and transferred to a phosphate buffer supporting electrolyte solution. Voltammograms were recorded between +0.3 and $+1.3 \mathrm{~V}$ vs. $\mathrm{Ag} \mid \mathrm{AgCl}$.

All the experimental curves presented were background-subtracted and baseline corrected using the moving average application with a step window of $1 \mathrm{mV}$ included in GPES version 4.9 software. This mathematical treatment improves the visualisation and identification of peaks over the baseline without introducing any artefact, although the peak intensity is in some cases reduced $(<10 \%)$ relative to that of the untreated curve. Despite this, this mathematical treatment of the original 
voltammograms was used in the presentation of all experimental voltammograms for a better and clearer identification of the peaks. The values for peak current presented in all graphs were determined from the original untreated voltammograms.

\section{Results and discussion}

\subsection{Voltammetry of polyadenosine ODN lipoplexes}

The differential pulse voltammograms obtained for the free ODN-I (Table 1), a homo-oligonucleotide composed only of adenosine nucleotides, and for the corresponding lipoplex with $(+/-)$ charge ratio of $1 / 1$ is shown in Fig. 1. It was found that the peak A1 at $+1.15 \mathrm{~V}$, due to adenosine nucleotide oxidation present in the free ODN-I, disappeared completely in the voltammogram obtained with the lipoplex, and that a new peak at $+0.60 \mathrm{~V}$ occurred as the only voltammetric peak, Fig. 1(a). This new peak is designated as peak L. As ODN-I contains only adenines, the peak L observed with the ODN-I lipoplex must be due to oxidation of adenosine nucleotides. An interesting point is the fact that the appearance of peak $\mathrm{L}$ is accompanied by a decrease in peak A and occurs for lipoplexes of (+/-) charge ratios $\geqslant 1 / 1$, which implies that the electrode

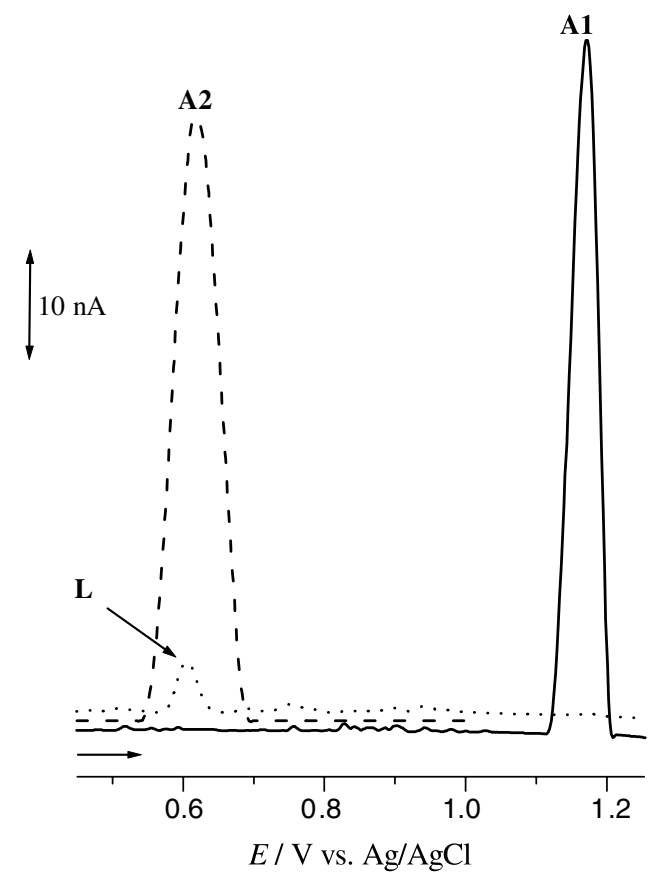

Fig. 1. Base line corrected differential pulse voltammograms obtained for: (-) ODN-I and ( ...) ODN-I lipoplex in pH 7.1 0.1 M phosphate buffer; (---) ODN-I in pH $120.1 \mathrm{M} \mathrm{NaOH}+\mathrm{KCl}$ electrolyte solution. Adsorption at $-0.1 \mathrm{~V}$ for $300 \mathrm{~s}$ was carried out before transferring the electrode for measurement in electrolyte solution. Pulse amplitude 50 $\mathrm{mV}$, pulse width $70 \mathrm{~ms}$, scan rate $5 \mathrm{mV} \mathrm{s}^{-1}$. L, lipoplex; A1, adenosine at $\mathrm{pH} 7.1 ; \mathrm{A} 2$, adenosine at $\mathrm{pH} 12$. process reflects an interaction between adenosine nucleotides and the cationic lipid DOTAP that compose the liposomes.

A model describing a possible reorganisation of lipoplexes at the electrode surface|solution interface during adsorption and voltammetric scanning was proposed where the electrostatic interactions between ODN lipoplexes and the electrode surface were assumed to play an essential role in the adsorption, Scheme 2. This model suggests the possibility of formation of a sandwich-like ODN-lipoplex configuration in which, for higher lipoplex $(+/-)$ charge ratios, a fraction of the ODN would stay at the interface between the electrode surface and the first lipid layer, and the rest of the ODN would be completely surrounded by liposomes. The electrode surface is thus covered by a network formed by the liposomes and the ODN in the ODN-lipoplexes close to the electrode surface which will sense a different $\mathrm{pH}$ environment from the bulk solution ODN reaching the uncovered electrode surface. However, only a small fraction of ODN would be close to the electrode surface, the rest of the ODN would be completely surrounded by liposomes, which explains the small intensity observed for peak L, Fig. 1.

The potential at which the oxidation of DNA bases occurs depends on the $\mathrm{pH}$ of the electrolyte buffer solution, since the potentials for purine base oxidation follow a $60 \mathrm{mV}$ per $\mathrm{pH}$ unit dependence [13]. Therefore, the higher the $\mathrm{pH}$ of the electrolyte buffered solution, the less positive is the potential necessary for guanosine or adenosine oxidation and the same is valid for their corresponding nucleotides.

To test the hypothesis that peak $\mathrm{L}$ could be due to adenosine oxidation in ODN-I in a different $\mathrm{pH}$ environment, it was estimated that a high $\mathrm{pH}$ would be necessary to shift the adenosine oxidation peak to $+0.60 \mathrm{~V}$.

A solution with the same concentration of ODN-I was prepared in $\mathrm{pH} 120.1 \mathrm{M} \mathrm{NaOH}+\mathrm{KCl}$ buffer electrolyte solution and a voltammogram was recorded in the same potential window $(+0.3$ to $+1.3 \mathrm{~V})$ after the mini-electrode with adsorbed ODN-I was transferred to the new electrolyte solution. The voltammogram obtained at $\mathrm{pH} 12$ for ODN-I, Fig. 1, shows only one peak at $+0.61 \mathrm{~V}$ corresponding to the adenosine oxidation at this $\mathrm{pH}$, peak $\mathrm{A} 2$.

The peak for adenosine oxidation was theoretically expected to shift $0.30 \mathrm{~V}$ towards less positive potentials with an increase of 5 units of $\mathrm{pH}$, but instead a change of $0.60 \mathrm{~V}$ was observed, peak A2. This discrepancy can be explained considering the effect of the electrode surface pretreatment that facilitates interaction between adenine nucleotides with glassy carbon surface groups, this being enhanced in alkaline electrolyte solutions. In fact, it was observed that the peak potential dependence of $\mathrm{pH}$ for adenosine in ODN-I is higher than $100 \mathrm{mV}$ in the $\mathrm{pH}$ range between 10 and 12 when the electrode 
surface was pretreated. This is in agreement with the fact that peak A2 has almost the same current but is wider at half height than peak A1 obtained in $\mathrm{pH}$ 7.1.

This demonstrates that the liposomes generate a micro environment with the very high $\mathrm{pH}$ responsible for the adenosines in the ODN-lipoplexes being oxidised at a potential of $+0.60 \mathrm{~V}$, peak $\mathrm{L}$, whereas the adenosines in the ODN will be oxidised at a potential corresponding to the $\mathrm{pH}$ of the bulk solution, Fig. 1(a).

\subsection{Effect of guanosine and adenosine nucleotides on ODN lipoplex voltammetry}

The differential pulse voltammograms obtained for ODN-II and for the coresponding ODN-lipoplex with a $(+/-)$ charge ratio of $1 / 1$, Fig. 2, present oxidation peaks corresponding to guanosine at $+0.90 \mathrm{~V}$, peak $(\mathrm{G})$, to adenosine at $+1.15 \mathrm{~V}$, peak (A) in the single strand [14-17] and to adenosine in the lipoplexes at $+0.60 \mathrm{~V}$, peak L.

It can be noticed for ODN-II that the peak current due to adenosine oxidation is higher than that for guanosine which can be correlated with the higher content in adenosine (6-A) compared to guanosine (4-G) in ODN-II. Furthermore, the ratios of 1.3 found for $I_{\mathrm{pA}} /$ $I_{\mathrm{pG}}$ or for the area under the peaks are approximately equal to the ODN-II base composition ratio of 1.5 found for $\mathrm{A} / \mathrm{G}$. It is reasonable to consider that this difference also reflects the base sequence, and this result is a good example of the potentiality of voltammetry for

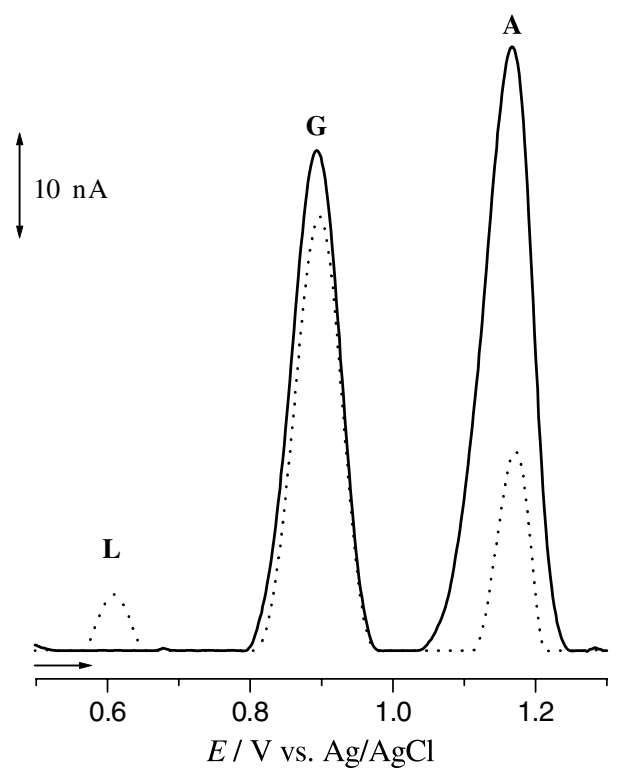

Fig. 2. Base line corrected differential pulse voltammograms obtained in $\mathrm{pH} 7.10 .1 \mathrm{M}$ phosphate buffer electrolyte solution for: (-) ODN-II and ( ...) ODN-II lipoplex (+/-) charge ratio 1/1. Adsorption at $-0.1 \mathrm{~V}$ for $300 \mathrm{~s}$ was carried out before transferring the electrode for measurement in electrolyte solution. Pulse amplitude $50 \mathrm{mV}$, pulse width $70 \mathrm{~ms}$, scan rate $5 \mathrm{mV} \mathrm{s}^{-1}$. L, lipoplex; G, guanosine; $\mathrm{A}$, adenosine. semi-quantitative and qualitative analysis of the relative ODN content in purines.

The peak $G$ in the ODN-II lipoplex shows a small decrease in current compared with the current obtained with free ODN-II. A very significant decrease in the current was recorded for peak A, and a new peak at $+0.60 \mathrm{~V}$ occurred for the ODN-II lipoplex, Fig. 2. This new peak has been identified as peak $\mathrm{L}$ and corresponds, as demonstrated in the previous section, Fig. 1, to the oxidation of adenosine in the ODN-II lipoplexes.

The current decrease in peak A for the ODN-II lipoplex in Fig. 2 corresponds to a decrease in the amount of free ODN adenosines close to the electrode surface. As shown in Fig. 1, the adenosine nucleotides in the lipoplexes encounter a chemical environment that is very deprotonated, generating a very high $\mathrm{pH}$, and their oxidation, peak L, occurs at the oxidation potential for adenosine at $\mathrm{pH}$ 12. The detection of peak A, Fig. 2, is due to the destabilising effect caused by the presence of guanosines which diminish the interaction between the ODN adenosines and the cationic lipid, causing some adenosine nucleotides not to sense the very local $\mathrm{pH}$ increase and to undergo oxidation at the bulk $\mathrm{pH}$ of 7.1.

The effect of the ODN-II lipoplex (+/-) charge ratio on the intensity of each of the three peaks mentioned, Fig. 3, shows that peak $G$ is characterised by an increase in the guanosine nucleotide peak current for a $(+/-)$ charge ratio between 0 and $1 / 2$, followed by a slow decrease with the rise in the cationic lipid content, in a similar way to what was previously observed for ODNs composed only of guanosine and cytidine nucleotides [10].

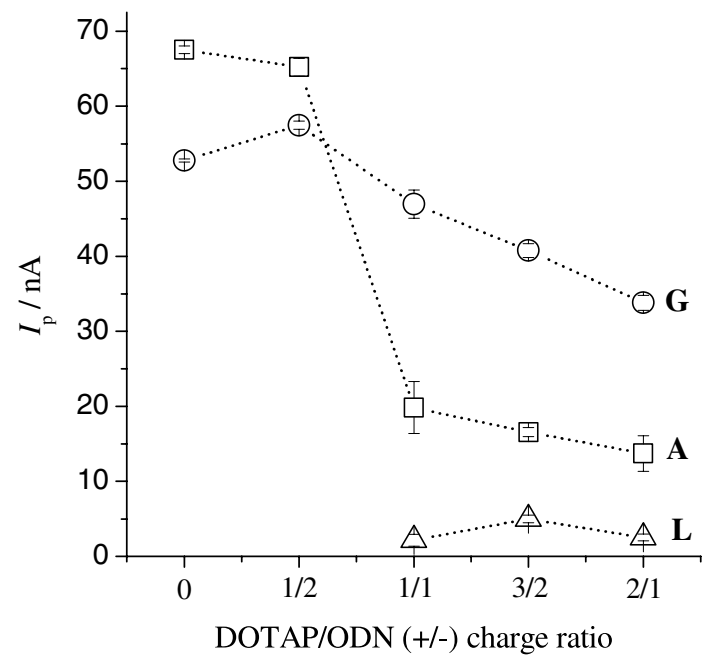

Fig. 3. Experimental relation found for $I_{\mathrm{p}}$ vs. ODN-II lipoplex (+/-) charge ratio for: $(\bigcirc)$ peak $G,(\square)$ peak $A$ and $(\triangle)$ peak L. An adsorption potential of $-0.1 \mathrm{~V}$ was applied for $300 \mathrm{~s}$. Each error bar represents the standard deviation obtained for $n=3$ experiments. L, lipoplex; G, guanosine; A, adenosine. 
On the other hand, the values found for the adenosine nucleotide peak current first show a small decrease until a $(+/-)$ charge ratio of $1 / 2$, followed by a sudden 3 -fold decrease between charge ratios $1 / 2$ and $1 / 1$. For higher charge ratios, a continuously smaller decrease of peak A was observed with a slope similar to that obtained between charge ratios 0 and $1 / 2$. The sudden current drop of peak A was accompanied by the appearance of peak L which occurred for a ODN-II lipoplex $(+/-)$ charge ratio $\geqslant 1 / 1$ and no great variation in current was observed for peak $\mathrm{L}$ with increase of lipid content.

\subsection{Effect of thymidine and cytidine nucleotides on $O D N$ lipoplex voltammetry}

The voltammograms obtained for ODN-III and for lipoplexes with a (+/-) charge ratio of $1 / 1$ obtained by mixing DOTAP liposomes and ODN-III are presented in Fig. 4. The voltammograms correspond to the curves obtained after moving the average base line correction of the original raw voltammetric data presented in the inset. As can be seen, no faradaic signal was detected over the entire potential window for free ODN-III or for the ODN-III lipoplex. This was expected for free ODN since the oxidation of the thymidine and cytidine nucleotides in ODN can be detected only at high positive
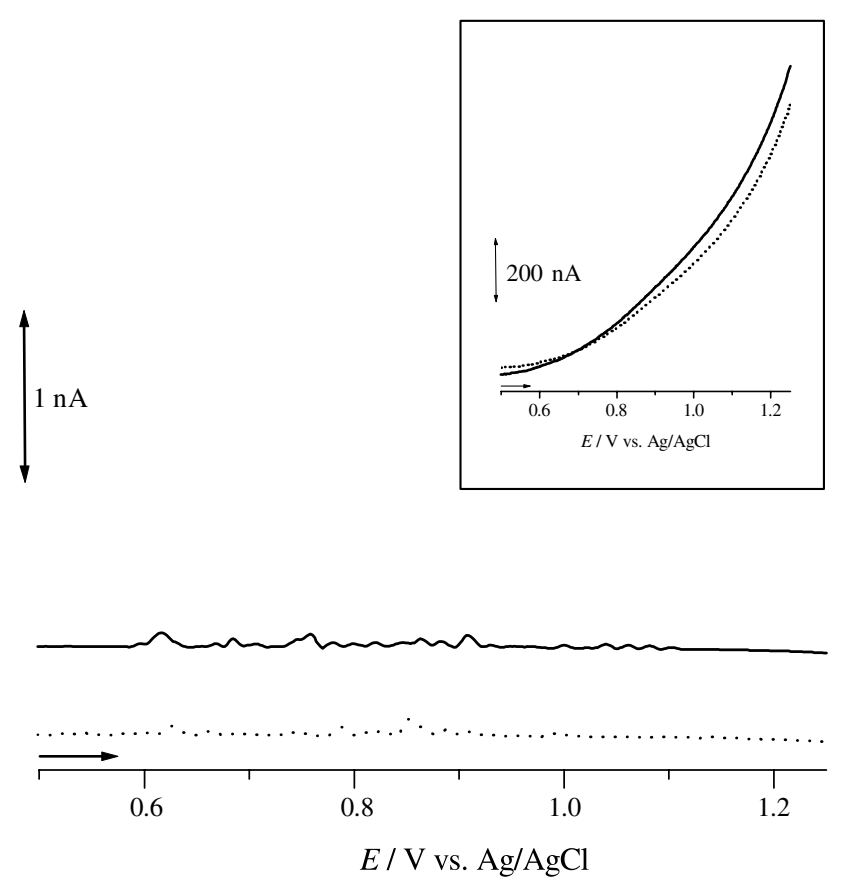

Fig. 4. Base line corrected differential pulse voltammograms obtained in $\mathrm{pH} 7.10 .1 \mathrm{M}$ phosphate buffer electrolyte solution for: (-) ODNIII and (...) ODN-III lipoplex (+/-) charge ratio 1/1. Inset: raw voltammograms. Adsorption at $-0.1 \mathrm{~V}$ for $300 \mathrm{~s}$ was carried out before transferring the electrode for measurement in electrolyte solution. Pulse amplitude $50 \mathrm{mV}$, pulse width $70 \mathrm{~ms}$, scan rate $5 \mathrm{mV} \mathrm{s}^{-1}$. potential, for the $\mathrm{pH}$ used, and their peaks are usually masked by the electrolyte oxidation [14-17]. The absence of signals for the ODN-III lipoplex shows, as predicted [16], that the presence of thymidine and cytidine [10], in the ODN sequence gave no detectable electrochemical signals.

\subsection{Voltammetry of ODN lipoplex with a genomic sequence}

The results obtained with short ODN sequences composed only of guanosine and adenosine nucleotides, Fig. 2, could also be observed with longer sequences containing all four DNA bases. The same voltammetric study was done with ODN lipoplexes of (+/-) charge ratio 1/1 using the 19-mer SALM-ODN and COMPODN (Table 1).

The SALM sequence is very interesting because it is characteristic of a specific fragment of Salmonella typhimurium gene, and COMP is its complementary sequence.

The voltammograms recorded in $\mathrm{pH} \quad 7.10 .1 \mathrm{M}$ phosphate buffer electrolyte solution for SALM-ODN, COMP-ODN and corresponding lipoplexes of $(+/-)$ charge ratio $1 / 1$, Fig. 5, present three oxidation peaks corresponding to guanosine at $+0.90 \mathrm{~V}$, peak $\mathrm{G}$, adenosine at $+1.15 \mathrm{~V}$, peak $\mathrm{A}$, and at $+0.67 \mathrm{~V}$, peak $\mathrm{L}$, in the lipoplex.

The ratio of the current and of the area between peak $\mathrm{G}$ and peak A for SALM-ODN was approximately proportional to the content in guanosine and adenosine bases in the ODN. Conversely and apparently in contradiction, the recorded voltammogram for COMPODN, Table 1, presents a more intense peak current for the oxidation of the guanosine nucleotides than that for the oxidation of the adenosine nucleotides although the content in adenosine (7-A) and guanosine (6-G) was similar in the ODN. There are two possible reasons. The first is the fact that COMP-ODN has three guanosine nucleotides at the $5^{\prime}$ end of the strand that can be oxidised more easily than those in the middle of the strand, which is the case for all the adenosine nucleotides. The second reason is linked to the possibility for COMPODN to form self-dimers and intra-strand structures such as hairpins that produce double helix regions hindering base oxidation because they are less exposed to the electrode surface. The analysis of the sequence COMP-ODN concerning its tendency to form self-dimers and hairpins enables the prediction of the formation of 3 self-dimers and 3 hairpins that, although not very stable, will have the three guanosine nucleotides at the $5^{\prime}$ end particularly exposed. These two reasons explain reasonably the apparently contradictory voltammetric results for the currents of the COMP-ODN peaks.

These data represent another example of the potential of voltammetry for semi-quantitative and qualitative 


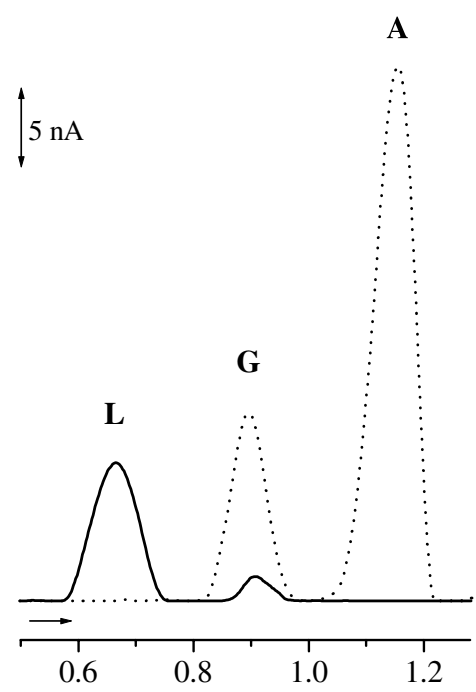

(a)

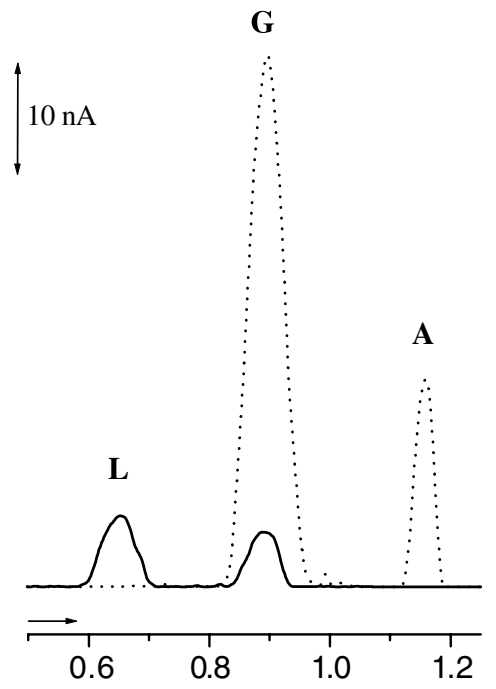

(b)

$E / \mathrm{V}$ vs. $\mathrm{Ag} / \mathrm{AgCl}$

Fig. 5. Base line corrected differential pulse voltammograms obtained in pH 7.1 0.1 M phosphate buffer electrolyte solution for: (a) ( $\cdots)$ SALM-ODN and (-) SALM-ODN lipoplex (+/-) charge ratio 1/1; (b) ( ...) COMP-ODN and (-) COMP-ODN lipoplex (+/-) charge ratio 1/1. Adsorption at $-0.1 \mathrm{~V}$ for $300 \mathrm{~s}$ was carried out before transferring the electrode for measurement in electrolyte solution. Pulse amplitude $50 \mathrm{mV}$, pulse width $70 \mathrm{~ms}$, scan rate $5 \mathrm{mV} \mathrm{s}^{-1}$. L, lipoplex; $\mathrm{G}$, guanosine; $\mathrm{A}$, adenosine.

analysis of the content in purines of a particular ODN and how it can be indicative of the presence of some motifs and structures.

In the voltammograms obtained for the corresponding ODN lipoplexes, Figs. 5(a) and (b), the absence of peak A was observed with a substantial depression in the current of peak $G$, and peak $L$ was shifted to $+0.67 \mathrm{~V}$. This change in the potential of peak L can be attributed to the presence of pyrimidines in the sequence and also to the greater length of these two ODNs, consequently with more negative charges per ODN strand. These two effects can contribute to different features in the complexation process [6-8]. The effect of the presence of pyrimidines in the ODN sequence did not cause any difference in the potential or shape of the purine peaks in the voltammograms.

Although a higher current reduction in peak $G$ with SALM and COMP-ODN lipoplexes was observed, Fig. 5, than for the ODN-II lipoplex, Fig. 2, a similar qualitative response was obtained.

\subsection{Voltammetric detection of denaturation of hybrid ODNs in lipoplexes}

Recent literature reported that double strand DNA suffers conformational transitions [18-21] and denaturation can occur [22] when mixed with cationic liposomes to generate lipoplexes of low $(+/-)$ charge ratio. By voltammetry, it is possible to distinguish between single strand and double helix DNA [14,15] since the peaks due to guanosine and adenosine nucleotide oxidation in DNA are comparably much higher and well defined for the single strand. In the double strand the bases are hindered inside the helix core, charge transfer to the electrode is more difficult, and normally no signal is observed in a first scan voltammogram $[14,15]$. This feature of DNA voltammetry was used to verify whether it is possible to sense voltammetrically a denaturation effect undergone by a double helix ODN hybrid inside the lipoplex. If the double helix of a hybrid ODN is unwound during the charge neutralisation that occurs with lipoplex formation, electrochemical detection of this change is possible by the appearance of any of the three peaks already identified and described in the previous sections for ODN lipoplexes.

In this context, the voltammetric response of lipoplexes formed with short duplexes obtained after hybridisation of the complementary sequences ODN-II/ ODN-III, and SALM-ODN/COMP-ODN were studied.

Voltammograms obtained for ODN-II, for the hybrid formed between ODN-II and ODN-III, and for the lipoplex formed by adding to the hybrid solution the amount of cationic liposomes necessary for a final $(+/-)$ charge ratio of $1 / 1$ are presented in Fig. 6 (see Section 2 for details on hybridisation). The voltammogram obtained for ODN-III is shown in Fig. 4 and, as mentioned, no peak is observed. The complete disappearance of peaks $A$ and $G$ from ODN-II after total hybridisation with ODN-I was confirmed, Fig. 6. Rigorously, it must be said that if there are some strands present that have not hybridised they are at concentrations that are not detectable by this technique (below $5 \mathrm{ng} / \mathrm{ml}[10])$.

When cationic liposomes were added to the hybrid solution in order to reach a final lipoplex with $(+/-)$ charge ratio of $1 / 1$, a peak was observed at $+0.90 \mathrm{~V}$, the 


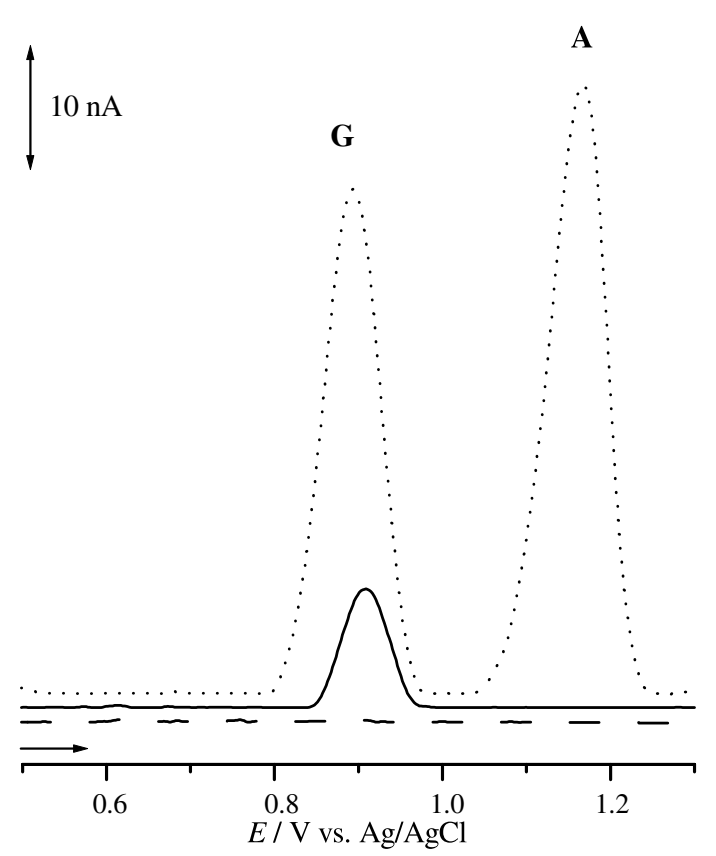

Fig. 6. Base line corrected differential pulse voltammograms obtained in $\mathrm{pH}$ 7.1 $0.1 \mathrm{M}$ phosphate buffer electrolyte solution for: ( ...) ODNII, (---) ODN-II/ODN-III hybrid, (-) hybrid lipoplex with (+/-) charge ratio of $1 / 1$. Adsorption at $-0.1 \mathrm{~V}$ for $300 \mathrm{~s}$ was carried out before transferring the electrode for measurement in electrolyte solution. Pulse amplitude $50 \mathrm{mV}$, pulse width $70 \mathrm{~ms}$, scan rate $5 \mathrm{mV} \mathrm{s}^{-1}$. G, guanosine; A, adenosine.

potential for oxidation of guanosine nucleotide, Fig. 6 . From the results presented earlier, this peak was attributed to the oxidation of guanosine nucleotides present in ODN-II, Fig. 2, used to make the hybrid, since for ODN-III no peaks were detected, Fig. 4. Peak A for adenosine nucleotide in ODN and peak L for adenosine nucleotide in the lipoplexes, already small in Fig. 2, are not detectable.

Since no peak was observed in Fig. 6 for the hybrid alone, it must be concluded that some double strands were unwound during lipoplex generation causing the appearance of peak $\mathrm{G}$ from guanosine nucleotides in the ODNs, which were exposed to the electrode surface. This peak current was lower than that recorded with the ODN-II alone which is an indication that not all the hybrids were unwound. In fact it is possible to estimate from the value of the peak current that at least $25 \%$ of the hybrid was unwound and was close to the electrode surface in such a way that the guanosine nucleotides could be oxidised.

The results obtained with the hybrid formed between SALM-ODN and its complementary sequence COMPODN with and without lipoplexes are presented in Fig. 7. These sequences are twice the length of ODN-II and ODN-III, besides the fact that they both have the four DNA bases in their composition. They are thus similar to genetic material normally used in genetic therapy using lipoplexes.

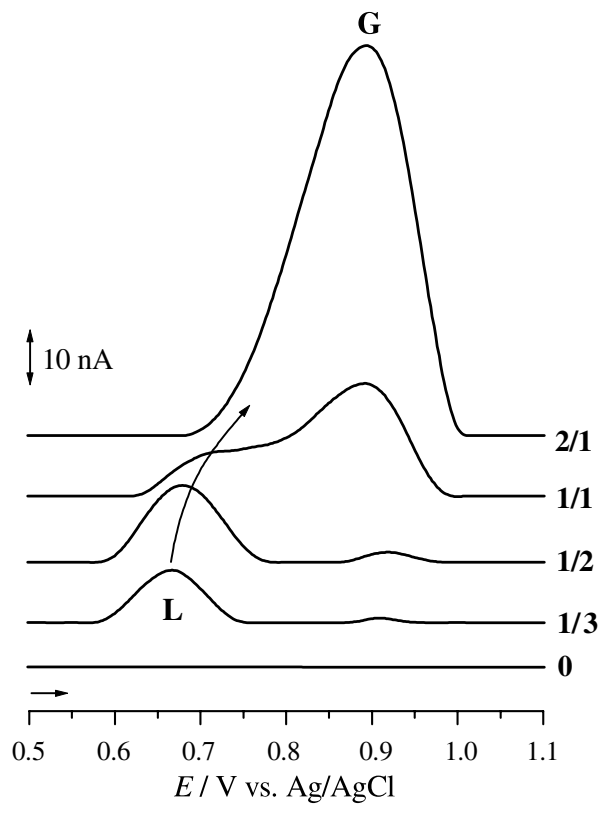

Fig. 7. Base line corrected differential pulse voltammograms obtained in $\mathrm{pH} 7.10 .1 \mathrm{M}$ phosphate buffer electrolyte solution for SALMODN/COMP-ODN hybrid lipoplex with different $(+/-)$ charge ratios. Adsorption at $-0.1 \mathrm{~V}$ for $300 \mathrm{~s}$ was carried out before transferring the electrode for measurement in electrolyte solution. Pulse amplitude 50 $\mathrm{mV}$, pulse width $70 \mathrm{~ms}$, scan rate $5 \mathrm{mV} \mathrm{s}^{-1}$. L, lipoplex; $\mathrm{G}$, guanosine.

As can be seen in Fig. 7, no peak was detected in the voltammogram recorded for the hybrid (charge ratio 0 ) indicating that all complementary ODN sequences have been hybridised. When the cationic liposomes were added to the hybrid, peak $L$ was seen as well as a very small peak G. The potential of peak L shifted progressively towards positive potentials as the lipoplex $(+/-)$ charge ratio increased (indicated by the curved arrow), and concomitantly peak $\mathrm{G}$ increased. For a (+/-) charge ratio of $2 / 1$, peak $G$ is so large that it includes the small and shifted peak L.

The behaviour reflected by the changes of peaks $\mathrm{L}$ and $\mathrm{G}$ with increasing lipoplex (+/-) charge ratio constitutes strong evidence that the double helix of the hybrid was partially unwounded during electrostatic interaction with the DOTAP liposomes. Furthermore, the observed data also suggest that the increase in the amount of liposomes, while the hybrid concentration was always maintained constant, caused the denaturation of an increasing number of hybrids. The single strand ODN formed became surrounded by cationic lipids forming single strand lipoplexes. The increase of peak $G$ current reflects the fact that more guanosine nucleotides from both ODNs are able to undergo oxidation and this is facilitated in the single strand form. Another interesting observation was the fact, that with a 6 -fold increase in the lipoplex (+/-) charge ratio, peak L which first appeared at $+0.67 \mathrm{~V}$ was shifted positively by at least $100 \mathrm{mV}$. 


\subsection{Discussion}

The voltammetric data obtained with ODN lipoplexes are very interesting for the characterisation and improvement of these gene delivery vectors and give insight into the possibility of the genetic material composing the lipoplex being able to be oxidised at charged interfaces. Indeed, it was found that guanosine nucleotides in ODN lipoplexes undergo oxidation at the same potential at which they are oxidised in free ODN. This is crucial, as it is known that guanosine is the most easily oxidised base in DNA [14,15,17] and that its principal oxidation product, 8-oxoguanosine [23], is mutagenic. This implies that even if the lipoplex formulation succeeds, in gene delivery there is a possibility that some of the bases composing the DNA could be oxidised during its travel to reach the nucleus of the target cell.

The voltammetric behaviour of ODN in lipoplexes showed that it is possible to distinguish by voltammetry the composition of lipoplexes prepared with ODNs of different base composition. Furthermore, it was shown that the lipoplex $(+/-)$ charge ratio influenced the voltammetric response.

There is evidence pointing to changes in surface potential and $\mathrm{pH}$ at the lipid|water interface during lipoplex formation, and that these changes depend on ODN base composition [6,7]. Pyrimidine homo-oligonucleotides are more effective in charge neutralisation than polyadenosine oligonucleotides due to the bulkier purine rings that make the approach of the adenosine nucleotide phosphates towards DOTAP quaternary amine group more difficult [6]. This effect means that polyadenosine lipoplexes are more positively charged than polypyrimidine lipoplexes for the same calculated lipoplex (+/-) charge ratio and the $\mathrm{pH}$ at the lipid|water interface is higher for polyadenosine than for polypyrimidine lipoplexes [6].

The observed shift to more positive potentials of peak L when pyrimidine nucleotides were present, Figs. 5 and 7 , also suggested that the local environment around adenosine nucleotides (that is the base sequence) affects their oxidation potential, consistent with a decrease in $\mathrm{pH}$ caused by the greater charge neutralisation with pyrimidine nucleotides.

The fact that the peak potential for guanosine nucleotide oxidation was not shifted in the lipoplex can be explained if the base molar volume is considered. Indeed, the guanosine molar volume is greater than that of adenosine, due to an extra carbonyl group linked to the purine ring, and this could reduce charge neutralisation and cause the surface $\mathrm{pH}$ to be similar to that of bulk solution. This reduced charge neutralisation implies a reduced level of lipoplex aggregation [6,7] and so guanosine nucleotides are much more exposed to the electrode surface.
The base proximity to the electrode surface is determined by the degree of linearisation due to the stretch of the ODN during the electrostatic interaction with DOTAP liposomes. It was reported that DOTAP cationic liposomes form more stable lipoplexes with relaxed DNA structures when plasmidic DNA has been used [20] and some similar features of the lipoplex generation driving force may be connected with the present work.

The detection of voltammetric peak currents for hybrid ODN lipoplexes was clear evidence that the duplex was partially unwound within the lipoplex, Figs. 6 and 7. It was also of interest to observe an increase in peak current with increasing lipoplex (+/-) charge ratio suggesting that charge neutralisation during lipoplex generation led to increasing helix unwinding, which also happens with an increase in $\mathrm{pH}$. The partial denaturation observed can also be caused by the dehydration occurring during lipoplex generation, which increases with aggregation [7,18].

The voltammetric experiments presented demonstrated, at least for the sequences studied and for DOTAP liposomes, the potential use of voltammetry to sense the denaturation caused by interaction between short double strand ODN and DOTAP liposomes. The results are in agreement with spectroscopic and enzymatic data, reported recently, in which the detection of partial denaturation in plasmidic DNA lipoplexes by increased hyperchromicity and nuclease susceptibility [20], and other methodologies was described [6-10,17]. They strongly suggest that the ODN composition can affect both the electrostatic and aggregation processes that occur during an ODN cationic liposome complexation event, and consequently can influence the efficiency of lipoplex transfection capacity.

\section{Conclusions}

The voltammetric characterisation of lipoplexes showed that the ODN base composition influences some physicochemical properties of lipoplexes related to the electrostatic and aggregation states.

The results suggest the successful application of voltammetry to study the effect of ODN base composition on lipoplex behaviour at electrified interfaces and to detect denaturation caused by interaction between short double strand ODN and DOTAP liposomes. They also show that voltammetry is a promising tool to study and characterise lipoplexes and complement the usual techniques in unravelling the different influences of purine and pyrimidine bases on lipoplex stability and their behaviour at the electrode|solution interface in an interdisciplinary effort to understand their interaction with biologically charged membranes and their transfection efficacy. 


\section{Acknowledgements}

Financial support from Fundação para a Ciência e Tecnologia (FCT), Ph.D. Grants PRAXIS XXI/BD/ 6134/2001 (J.A.P.P.) and PRAXIS XXI/SFRH/BD/ 4897/2001 (M.M.), POCTI (co-financed by the European Community Fund FEDER), ICEMS (Research Unit 103) and European Projects ERBIC15-CT98-0915, QLK3-2000-01311 and HPRN-CT-2002-00186 are gratefully acknowledged.

\section{References}

[1] J.S. Cohen, Adv. Pharmacol. 25 (1994) 319.

[2] F. Shi, L. Wasungu, A. Nomden, M.C. Stuart, E. Polushkin, J.B. Engberts, D. Hoekstra, Biochem. J. 366 (2002) 333.

[3] O. Zelphati, F.C. Szoka Jr., Proc. Natl. Acad. Sci. USA 93 (1996) 11493.

[4] S. Audory, D. Hoekstra, Mol. Membr. Biol. 18 (2001) 129.

[5] S. Simões, P. Pires, N. Duzgunes, M.C. Predroso de Lima, Curr. Opin. Mol. Therapeutics 1 (1999) 147S.

[6] V.M. Meidan, J. Glezer, N. Amariglio, J.S. Cohen, Y. Barenholz, Biochim. Biophys. Acta 1568 (2001) 177.

[7] V.M. Meidan, J.S. Cohen, N. Amariglio, D. Hirsch-Lerner, Y. Barenholz, Biochim. Biophys. Acta 1464 (2000) 251.

[8] P. Jurkiewicz, A. Okruszek, M. Hof, M. Langner, Cell Mol. Biol. Lett. 8 (2003) 77
[9] M.E. Ferrari, D. Rusalov, J. Enas, C.J. Wheeler, Nucleic Acids Res. 29 (2001) 1539.

[10] J.A.P. Piedade, M. Mano, M.C. Pedroso de Lima, T.S. Oretskaya, A.M. Oliveira-Brett, (submitted).

[11] S. Simões, V. Slepushkin, R. Gaspar, M.C. Pedroso de Lima, N. Düzgünes, Gene Ther. 5 (1998) 955.

[12] H.-P. Dai, K.-K. Shiu, J. Electroanal. Chem. 419 (1996) 7.

[13] G. Dryhurst, F.J. Elving, J. Electrochem. Soc. 115 (1968) 1014.

[14] C.M.A. Brett, A.M.O. Brett, S.H.P. Serrano, J. Electroanal. Chem. 366 (1994) 225.

[15] A.M. Oliveira-Brett, S.H.P. Serrano, J.A.P. Piedade, in: R.G. Compton, G. Hancock (Eds.), Comprehensive Chemical Kinetics, vol. 37, Elsevier, Amsterdam, 1999, p. 91 (Chapter 3).

[16] A.M. Oliveira Brett, F.-M. Matysik, J. Electroanal. Chem. 429 (1997) 95.

[17] A.M. Oliveira Brett, F.-M. Matysik, Bioelectrochem. Bioenerg. 42 (1997) 111

[18] S. Choosakoonkriang, C.M. Wiethoff, G.S. Koe, T.J. Anchordoquy, C.R. Middaugh, J. Pharm. Sci. 92 (2003) 115.

[19] W. Huang, Z. Zhang, X. Han, J. Tang, J. Wang, S. Dong, E. Wang, Bioelectrochemistry 59 (2003) 27.

[20] C.M. Wiethoff, M.L. Gill, G.S. Koe, J.G. Koe, C.R. Middaugh, J. Biol. Chem. 277 (2002) 44987.

[21] S. Even-Chen, Y. Barenholz, Biochim. Biophys. Acta 1509 (2000) 188.

[22] T.K. Prasad, V. Gopal, N.M. Rao, Biochim. Biophys. Acta 1619 (2003) 69.

[23] A.M. Oliveira Brett, J.A.P. Piedade, S.H.P. Serrano, Electroanalysis 12 (2000) 973. 